\title{
Correlation of Accelerometer and Microphone Data in the "Coin Tap Test"
}

\author{
Huadong Wu, Mel Siegel \\ Robotics Institute, Carnegie Mellon University
}

\begin{abstract}
The "coin tap test" is one of the oldest methods of nondestructive test (NDT). It requires an operator to tap with a coin-like light tool on each point of the structure to be inspected, feeling the subtle difference of impact force and hearing the resulting sound to discriminate defective objects from normal ones. Although this is the one of the most cost-effective NDT methods for detection of delamination in multi-layered materials, the test technology still remains largely subjective, and there has been considerably uncertainty about the physical principles behind it. Through analyzing and comparing the different aspects of the impact in the coin-tap test mainly the force measured by an accelerometer in the hammer and the resulting sound recorded with a microphone - this paper seeks a better understanding of the fundamental principles underlying the individual measurement techniques, and it gives a paradigm for sensor fusion via using the data from one modality to select the optimal time window for signal analysis of another modality.
\end{abstract}

\subsection{Introduction}

The "coin tap test" (or "screwdriver handle test") is a venerable means for manually verifying the integrity of objects and structures, particularly sheet-like and layered materials that are subject to cracking and delamination. It requires an operator to tap with a small hammer (or a screwdriver handle or some other light-weight object, like a coin) each point of the structure to be inspected, meanwhile feeling the rebound of the hammer and listening to the resulting sound radiated by the impact. Healthy examples typically reverberate cleanly (they sound "live"), whereas damaged examples yield a sound that is dull ("dead"). The operator can discriminate defective examples from good ones by discerning the differences.

The coin-tap method is distinguished from superficially similar techniques that excite modal resonances, as in the hammer testing of railway carriage wheels (which ring like bells when properly struck), in that it delivers a smaller impulse, so that only the local structure is excited. The sound then depends on local mechanical characteris- tics such as mechanical elasticity and impedance etc.

There are essentially four techniques in the family of local methods. In addition to the coin-tap test, these are the mechanical impedance method, the membrane resonance method and the velocimetric method. The mechanical impedance method measures the resistance to motion of the local structure given the applied force; the membrane resonance method measures the fundamental resonance frequency under wide-band excitation; the velocimetric method is similar to the membrane method but the exciter and the transducer (receiver) are set farther apart making it sensitive primarily to the structure flexing (bending) modes.

All these local impact NDT methods basically use direct mechanical impacts as exciting sources, and all measure only the responses of the impacts within the human audible sonic frequency range, from around $20 \mathrm{~Hz}$ up to around $20 \mathrm{kHz}$. Thus they are also categorized as low-frequency NDT methods, versus ultrasonic methods.

The traditional coin-tap test is subjective, that is, its effective implementation depends on the operator's skill and experience. If we carefully study how an operator implements this technique, it seems that there are three important aspects: (1). how large an impulse is suitable to make the response "local" and distinctive; (2). how to analyze subtleties in the resulting sound; (3). how to analyze the enhanced tactile response.

Though the coin-tap test seems very simple, its response varies vastly according to the structure excitation extent. Physically complex questions remain to be satisfactorily answered, for example, how "locally" the impact should be applied and the measurements taken, what is the relationship between impulse magnitude and the resulting sound amplitude and spectrum composition, etc. In this paper, we begin our investigation with impact analysis, then look into hammer acceleration data and sound data, trying to build up a sensor fusion paradigm to improve the coin-tap NDT technology.

\subsection{Impact Analysis in Coin-tap Test}

As the coin-tap technique deals with the local excitation behavior within a global structure, it is very difficult 
to model. There have been only sporadic research activities in this area, and the results are incomplete. We now seek the basic mechanical relationship between the contact force profile and the structure properties.

The classical theory of impact, primarily based on the impulse-momentum law for rigid bodies - assuming that the kinetic energy transformed into the body's vibration is negligible - is incapable of describing the transient forces, stress, or the deformations produced; thus it cannot explain the interaction force profile and the "ring" we hear. The analysis of impact and vibration requires elasticity and plasticity models, which generally do not yield a closed-form solution.

Following the wave motion theory in elastic solids articulated by Goldsmith in the 1950s [4], Cawley numerically simulated a few impact cases between a light hammer and a free-free beam [5]. Figure 1 illustrates how a typical interaction force profile (the solid curve) would look.

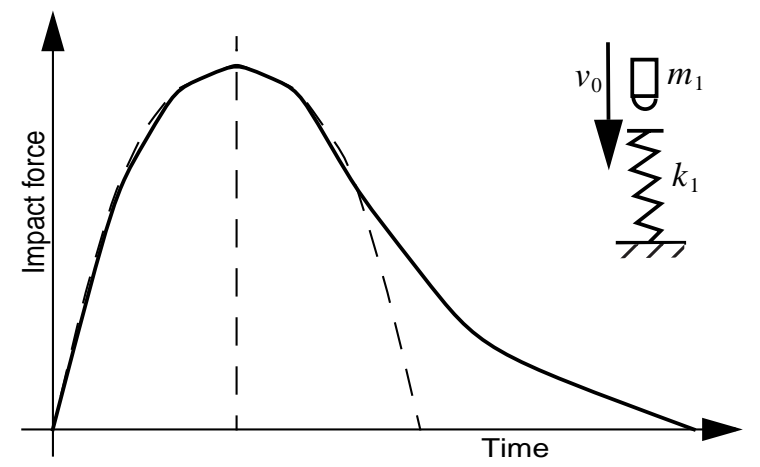

\section{FIGURE 1. Expected force-time curve for thin structure}

It is notable that the force-time history curves are asymmetrical with respect to the peak: the duration after the peak is significantly longer than the rise time to the peak. This "tail" on the impact force profile is due to the vibration of the beam. It is more severe with a thinner beam, both because the vibration amplitude in a particular mode excited by a given force is increased as the stiffness of the beam is reduced, and also because more modes come into the frequency range which is excited by the impact.

This analysis begins revealing the physical complexity of the coin-tap tests. In fact, severe non-linearity comes not only from the change of mode number (as shown the above analysis), but also from the variation of energy dispersion or attenuation with respect to the impact force magnitude.

All currently reported coin-tap research avoids these mathematically enormous difficulties by approximating the hammer impact process by a half-cycle sine vibration.
In this simplified model, the hammer (mass $m_{1}$ ) is attached to the ground via a simple spring (with stiffness $k_{1}$ ) as shown in upper-right corner of Figure 1.

For a single clean impact, to first order the contact time is independent of impact velocity and depends only on the hammer mass $m_{1}$ and the surface contact stiffness $k_{1}$; the hammer rebounds briskly from an intact surface and sluggishly from a damaged surface ${ }^{1}$ as the effective $k_{1}$ is reduced when the material weakens.

The contact time $T_{1}$ is just a half cycle of the massspring oscillation:

$$
T_{1}=\pi \cdot \sqrt{m_{1} / k_{1}}
$$

This force (or acceleration) versus time relation is a half-cycle sine curve shown in Figure 1 as a dashed line.

\subsection{Available Products and Our Experiments}

\subsection{Commercially Available Products}

Based on the above described single-spring model, several commercial instrument systems have appeared. The most popular, at least in the aircraft inspection application that we know best, are the Mitsui "Woodpecker" [1] (advocated by Airbus for nondestructive testing of composite laminated aircraft skin components) and the WichiTech "RD3" instrumented hammer [2] (a commercial version of apparatus developed by Georgeson et al at Boeing [3]). The Mitsui product uses a solenoid-driven hammer and the WichiTech product uses a hand-wielded hammer; however both instruments measure essentially the output of an accelerometer embedded in the hammer head.

Basically both instruments base their judgements on just the contact time duration referenced to a normal sample; however in Mitsui's patent document [8] a method of using the force/acceleration-time history asymmetry measurement was also mentioned.

Another commercially available coin-tap test instrument we know of is Rolls-Royce's "MetEval Tapometer". It measures the output of a force gauge mounted on the head of the small solenoid-driven hammer. Also with referenced to samples believed to be normal, the test judgement is based on contact time duration and/or the

1. This first order (harmonic oscillator) model has been found to be valid in other analogous contexts, e.g., the contact time of a tennis ball on the racket or the ground depends on the state of the ball ("live" or "dead") and the nature of the surface off which it bounces, but hardly at all on the impact velocity [7]. 
frequency spectrum of the recorded force-time history.

An alternative way of implementing the traditional coin-tap test is to analyze the impact-generated sound data instead of the force data. Indeed, we believe that a human inspector uses primarily the sound, although it is at least plausible that s/he also derives useful information from the haptic/tactile quality of the hammer blow.

Bruce Pfund of SP Surveys is a proponent of the anthropomorphic approach. His "Smart Hammer Systems" employs a pneumatically driven hammer, a microphone coupled to the hammer impact through the air, and graphical display of the acoustic Fourier spectrum to help the inspector decipher the anvil's condition [9].

Pfund argues that in complex real world environments, with surfaces in arbitrary orientations and states of contamination, the sound per se, propagated through the air, is a better indicator of subsurface condition.

To investigate whether one approach is better than the other under similar conditions in terms of sensitivity and reliability, we did sound data analyses and compared the results with those of force analysis. The sound data were recorded from the identical impacts as in the experiments described below.

\subsection{Our Experiments}

Our goal is to improve our understanding of the fundamental principles underlying coin-tap test techniques, and to evaluate how the commonly used measurement paradigm works in commercially available products.

3.2.1 Experimental Equipments. The experimental system is illustrated in Figure 2.

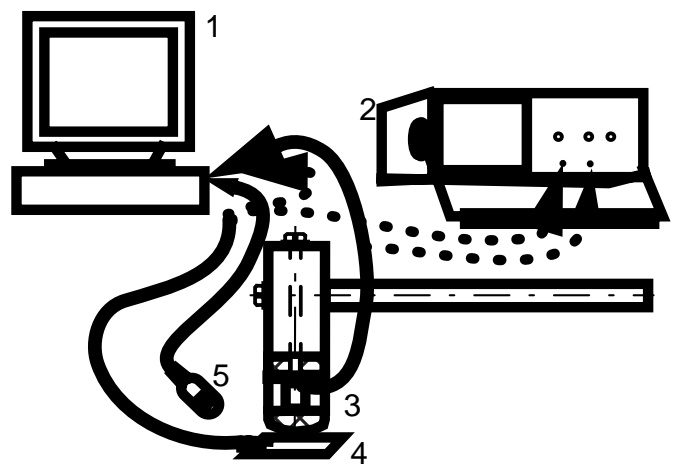

FIGURE 2. Test system.

The apparatus we used includes: an SGI Indy multimedia workstation (1) with dual-channel audio sampling capability; a Tektronics 2232 dual-channel digital sampling oscilloscope (2); a small hammer with various material and head weights, inside each of which we can mount a Kristler 811AD accelerometer (3); microphones (5); and Kynar piezoelectric film (4) (Atochem North America's PVDF) for detecting vibrations transmitted through the sample material surface.

The Tektronics 2232 dual-channel oscilloscope has the capability of simultaneously storing and displaying signals with proper single-sweep trigger control. We mainly used this feature to study force-time history curve profile shape variations.

Trying to better understand different aspects of the coin-tap process, we experimentally compared steel versus plastic hammers' behaviors, as well as piezoelectric film and accelerometer data. The scope of this paper is restricted to results using a plastic hammer (30 gram) to tap on an airplane skin.

3.2.2 Data Collection. To evaluate the effectiveness of the currently most popular coin-tap methods and to seek possible improvements, we compare the test results on patched versus normal airplane skin, with different underskin structure conditions. We name two typical types of under-skin structure conditions: condition $A$ as those points close to some supporting joists and condition $B$ as those points relatively far away from any supporting components.

Through the dual-channel audio input port of the SGI Indy workstation, we recorded both the acceleration and corresponding sound data simultaneously, of some chosen typical normal airplane skin and typical patched skin, with under-skin structure condition $\mathrm{A}$ and condition $\mathrm{B}$ respectively. The sampling rate is $48 \mathrm{kHz}$

3.2.3 Basic Data Analysis Algorithm. With the recorded force and sound data, we first measure the contact time duration $T_{1}$ between the hammer and the skin. A typical acceleration-time history curve is asymmetric in shape with notable noise, as shown in Figure 3.

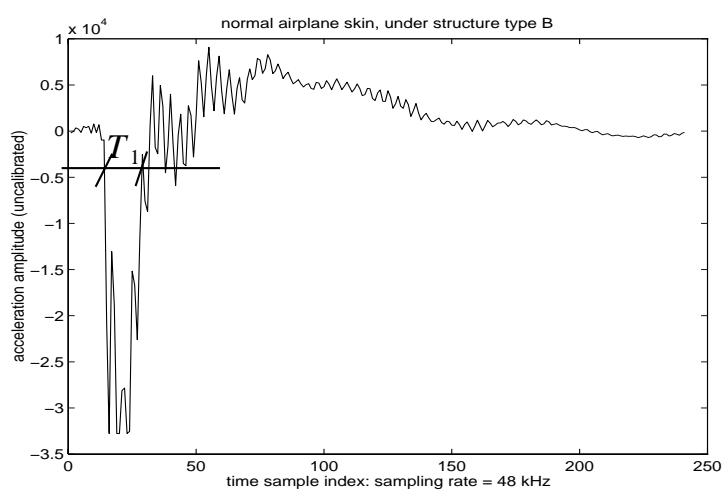

FIGURE 3. Typical hammer acceleration history. 
To investigate the force and sound spectrum distributions, we calculate Cawley's [5] 1/3 power accumulation ratio factor $R_{1 / 3}$. The $1 / 3$ power accumulation ratio factor $R_{1 / 3}$ is defined as:

$$
R_{1 / 3}=\frac{\sum_{i=1}^{M / 3} \operatorname{Pxx}\left(f_{i}\right)}{\sum_{i=1}^{M} \operatorname{Pxx}\left(f_{i}\right)}
$$

where the $\operatorname{Pxx}\left(f_{i}\right)$ is the spectrum component at frequency $f_{i}$, and $M$ is the number of calculated frequency components. To keep our experiments consistent with Cawley's and the Mitsui people's work, we use the lower frequency components of up to $8 \mathrm{kHz}$.

For a typical under-skin structure condition type A and condition type B respectively, we conducted about 10 tests on patched and normal skin respectively. The following sections address these data analysis and interpretation.

\subsection{Acceleration Data Analysis}

A typical complete acceleration event lasts for less than 0.01 seconds in our airplane skin coin-tap inspection. To safely avoid losing useful information we took 512 points for the following analysis.

The data file begins with a quiet lead (36 points, or 0.75 milliseconds). As described in the last section, we first manually collected about impacts with similar contact duration $T_{1}$ values, calculated the mean and standard deviation $\sigma$ of the duration distribution. Then we did the Fourier analysis and calculated the 1/3 power accumulation ratio factor $R_{1 / 3} \mathrm{~s}$. The result is shown inTable 1

As the above data are sampled at $48 \mathrm{kHz}$ and 512 points were used to do the FFT analysis, the frequency granularity in spectrum analysis (calculating $R_{1 / 3}$ ) is about $94 \mathrm{~Hz}$.

In contradiction to the single-spring model, where in the unsupported case $k$ should be smaller and so the contact time should be longer, we observe a shorter contact time in the unsupported case. We speculate that this happens because in the supported case the impact is coupled to high frequency modes of the stiff under-structure.

\subsection{Sound Data Analysis}

While a typical complete acceleration event lasts for less than 0.01 seconds, the radiated sound in our airplane inspect tests lasts for about $0.15 \sim 0.2$ seconds for the same impacts. We took 2048 sound amplitude samples (over
TABLE 1. Contact time duration $T_{1}$ and $1 / 3$ power spectrum $R_{1 / 3}$

\begin{tabular}{|c|c|c|c|c|}
\hline & & & Normal skin & Patched skin \\
\hline \multirow{4}{*}{$T_{1}$} & \multirow{2}{*}{ supported } & mean & $0.6114 \mathrm{~ms}$ & $0.3282 \mathrm{~ms}$ \\
\hline & & $\sigma$ & $0.0152 \mathrm{~ms}$ & $0.0253 \mathrm{~ms}$ \\
\hline & \multirow{2}{*}{$\begin{array}{l}\text { un-sup- } \\
\text { ported }\end{array}$} & mean & $0.3856 \mathrm{~ms}$ & $0.3055 \mathrm{~ms}$ \\
\hline & & $\sigma$ & $0.0170 \mathrm{~ms}$ & $0.0314 \mathrm{~ms}$ \\
\hline \multirow{4}{*}{$R_{1 / 3}$} & \multirow{2}{*}{ supported } & mean & 0.3615 & 0.3595 \\
\hline & & $\sigma$ & 0.0025 & 0.0024 \\
\hline & \multirow{2}{*}{$\begin{array}{l}\text { unsup- } \\
\text { ported }\end{array}$} & mean & 0.3653 & 0.3526 \\
\hline & & $\sigma$ & 0.0019 & 0.0013 \\
\hline
\end{tabular}

$\sim 0.043$ seconds) for the following data analysis, so the granularity in frequency spectrum analysis is $23.4 \mathrm{~Hz}$.

From these sound samples (using the identical impacts we used in force analysis), we also calculated $1 / 3$ power accumulation ratio factor $R_{1 / 3}$, with frequency components from $23.4 \mathrm{~Hz}$ up to $8 \mathrm{kHz}$ (for comparison with Pfund's practice, $10 \mathrm{kHz}$ as shown in [10]). The mean and standard deviation $\sigma$ are shown in Table 2.

TABLE 2. Sound power spectrum $R_{1 / 3}$

\begin{tabular}{|c|c|c|c|}
\hline \multicolumn{2}{|c|}{$R_{1 / 3}$} & Normal skin & Patched skin \\
\hline \multirow{2}{*}{ supported } & mean & 0.3664 & 0.3703 \\
\cline { 2 - 4 } & $\sigma$ & 0.0029 & 0.0045 \\
\hline \multirow{2}{*}{ unsupported } & mean & 0.3735 & 0.3719 \\
\cline { 2 - 4 } & $\sigma$ & 0.0019 & 0.0015 \\
\hline
\end{tabular}

\subsection{Relationship between surface dynamics and emitted sound}

Comparing the results in the two tables, we notice that while the distributions of normal skin $R_{1 / 3}$ and patched skin $R_{1 / 3}$ have a large overlap for supported areas (underskin condition A) in Table 1, a very similar situation exists for the unsupported areas in Table 2. This means that the coin-tap test method, either with force measurement only or with sound measurement only, cannot always distinguish different airplane skin conditions for some underskin supporting structure conditions, and the discrimina- 
tion capabilities of the force-only method and sound-only method are very similar.

This also suggests that the two measurements actually contain similar information. However, a close examination of the impact data reveals some interesting points. First, as the interesting part of the force-time history is much shorter than the sound duration, there may be some additional potentially useful information in sound data, and this additional or redundant information may lead to more reliable defect detection when properly used. On the other hand, it is difficult to use the sound signal to deduce the impact amplitude, so the force-time history profile shape is still the best indicator both to the under-structure complexity and to the impact amplitude.

Coin-tap test reliability should be further improved if we can fuse these two sets of data. For example, a common case is that there are multiple interactions between the hammer and the tested surface in a nominally single tap. The force-time history is very sensitive to this situation, but then the curve will become so asymmetric that Cawley's single-spring model is no longer valid. Thus sound data would be more useful, although they are less sensitive. Figure 4 shows how the force-time history and the sound data behave in such a situation.
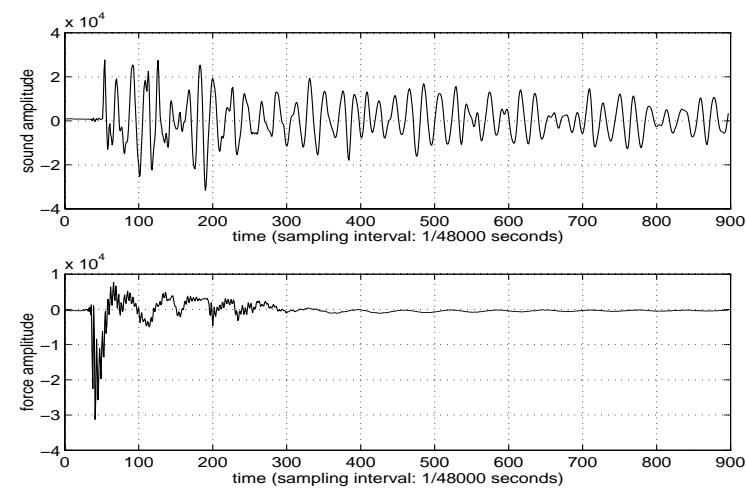

\section{FIGURE 4. Typical force profile and the corresponding sound amplitude showing a severe non-linearity in thin-surface structure test.}

A careful observation of the recorded data as in Figure 4 reveals that the sound pattern changes after the interaction ends completely. That the free-vibration part of the sound carries useful information is illustrated in Figure 5 where it is obvious that although the frequency spectra of force (a) and whole-sound waves (b) show noticeable distribution patterns, the frequency spectrum distribution of the free-vibration sound part (c) is much more unique and easier to distinguish.
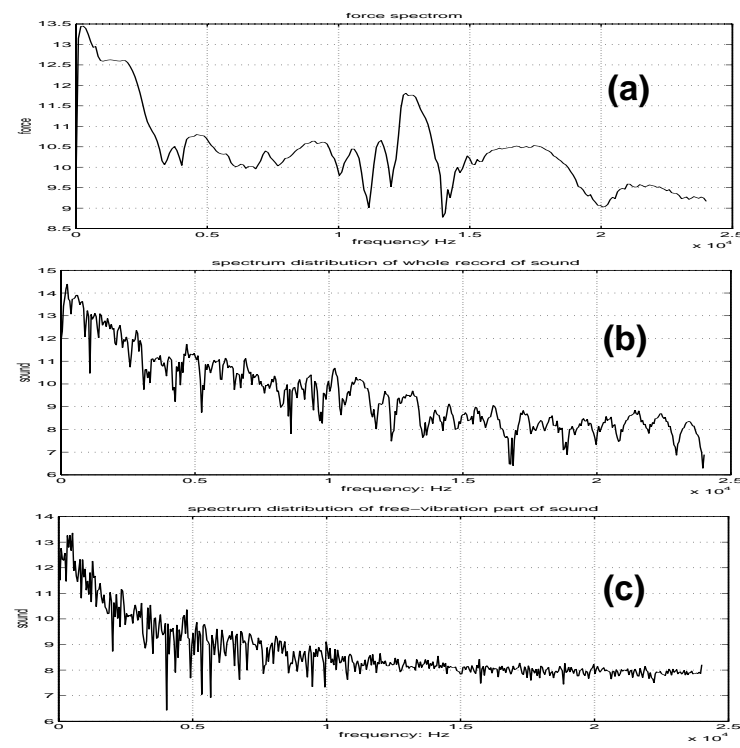

FIGURE 5. Frequency analysis of (a).force-time history(512 samples); (b).the whole sound amplitude history; (c).free-vibration part of the sound amplitude history

In Table 3 the 1/3 power accumulation ratio factors $R_{1 / 3}$ on points with different under-skin supporting structure (condition A and B) are calculated, according to Equation $(E Q 2)$ described in Section 3.2.3, from the freevibration part of the recorded sound samples.

TABLE 3. Sound power spectrum $R_{1 / 3}$ from freevibration, for different under-skin supporting structures

\begin{tabular}{|c|c|c|c|}
\hline \multicolumn{2}{|c|}{$R_{1 / 3}$} & Normal skin & Patched skin \\
\hline \multirow{2}{*}{ supported } & mean & 0.3795 & 0.3905 \\
\cline { 2 - 4 } & $\sigma$ & 0.0035 & 0.0045 \\
\hline \multirow{2}{*}{ unsupported } & mean & 0.3777 & 0.3892 \\
\cline { 2 - 4 } & $\sigma$ & 0.0027 & 0.0013 \\
\hline
\end{tabular}

Comparing the results of tables 1,2 and 3, it can be seen that Table 3 provides the clearest indication of both skin status and of under-skin supporting structures.

This suggests that we adopt a paradigm in which the force-time history data are used to decide whether the particular impact is good one for detecting a particular type of defects, and if it is, then to detect when the interaction between the hammer and surface ends - at this point the free-vibration part of sound begins, and it is this last part of the sound history that it is useful to analyze for specific defect and environment characterization. 


\subsection{Conclusion and Further Research}

Our survey of the literature of the field of coin-tapping test technology, the commercial products now on the market, the instruments being used in key applications (aircraft skins, boat hulls), and our own experimental results all tend to support our working hypothesis that both microphones and accelerometers have their separate valid roles as instrumentation suitable for automating defect detection. Further more, our research results show that by force and sound measurement sensor fusion it is possible to make "the whole greater than the sum of the parts".

Specifically our experiments lead us toward these conclusions:

- It is hard to say in any universal sense whether forceonly or sound-only methods are more useful coin-tap methods.

- When the surface being tested is very thin, especially when the under-surface supporting structure is also very complex, there are multiple interactions between the hammer and the surface, making the simple singlespring linear model an inappropriate representation of the impact process.

- The force-time history curve shape is a good indicator of whether a particular impact was of appropriate strength, and to locate the start of free-vibration part of the sound amplitude record.

- The free-vibration part of the sound amplitude record is more useful than either the whole record of sound amplitude or the force-time history data in detecting surface defects or under-surface structure differences.

- Based on limited data (e.g. Table 3), it seems that using this method we can conclusively discriminate patched vs. unpatched regions, and we may be able to discriminate, within each of these classes, supported vs. unsupported regions. Since the presence of absence of supporting structure is generally known from design drawings, etc., but the location of patches is rarely well documented, the approach seems to be practical value even at its present early stage.

We believe that patching and defect development will have similar effects on the signal, but this remains to be demonstrated with defect samples.

Because the impact energy deposited by a coin-tap undergoes dispersion and attenuation via excitation and propagation of multiple frequency modes, the relationship between the impact magnitude and the force-time history or the sound amplitude-time history is quite nonlinear. This makes it difficult or impossible to normalize each force-time or sound-amplitude-time history to the tap-totap velocity and delivered impulse variations.

To overcome this fundamental difficulty, our future research will examine learning methods that utilize a training set that is representative of a comprehensive range of sample types, hammer types, and impact delivery strategies.

\subsection{References}

1. Mitsui Engineering \& Shipbuilding Co., Ltd., Advanced Machine \& System Division, "Technical Report No.2 V1.1, Mitsui Woodpecker", 1993

\section{WichiTech, Columbia MD.}

3. Gary Georgeson, Scott Lea, and Jeff Hansen (Boeing Defense \& Space Group), Electronic tap hammer for composite damage assessment, Nondestructive Evaluation of Aging Aircraft, Airports, and Aerospace Hardware, SPIE Vol. 2945, Scottsdale AZ, 3-5 December 1996.

4. W. Goldsmith 1960, "Impact - The Theory and Physical Behavior of Colliding Solids", London: Edward Arnold.

5. P. Cawley and R. D. Adams, "The Mechanics of the Coin-Tap Method of Non-Destructive testing", Journal of Sound and Vibration, 1988, 122(2), 299 316

6. P. Cawley, "Low Frequency NDT Techniques for the Detection of Disbonds and Delaminations", British Journal of NDT, Vol.32, No.9, September 1990, page 454 461

7. Howard Brody, Tennis Science for Tennis Players, University of Pennsylvania Press, 1987.

8. U.S. Patent No. 05,048,320, "Method and Apparatus for Impact-type inspection of Structures", Kunihiro Mitsuhashi, Chihiro Jyomuta, Fujio Oka, and Hidetoshi Nishikawa, Sept. 17, 1991.

9. U.S. Patent No. 05,686,652, "Portable Test Hammer Apparatus", Bruce Pfund, Sept. 9, 1996.

10. Advertisement flyer "Smart Hammer Systems", Pfund. 